\title{
SUPLETIVISMO VERNÁCULO COMO PROFILAXIS DE LA PÉRDIDA DE TRANSPARENCIA DERIVACIONAL ¿QUÉ MILANESAS QUE TE DEJAS VERACRUZ POR ACAPULCO!
}

\begin{abstract}
A lo largo del presente siglo, la interacción entre fonología y morfología ha sido tema de estudio frecuente, desde los ajustes necesarios a las leyes de Grimm (Rask) y Verner, pasando por los trabajos de Trubetzkoy sobre morfofonología, los estudios detallados de romanistas como Wahlgren ${ }^{1}$ y Malkiel $^{2}$ hasta los de Martinet (1965) Stankiewicz (1962), Rochet (1974), Hooper (1976), Dressler (1977), Rudes (1980) y los de la Chicago Linguistic Society (1983) entre otros. Se han abordado diversos aspectos de esta interrelación, preferentemente en trabajos diacrónicos.
\end{abstract}

Se oponen por un lado la tendencia a la pérdida de identidad

${ }^{1}$ ERnst G. WAhlgren, Étude sur les actions analogiques réciproques du parfait et du participe passé dans les langues romanes, tesis, Uppsala 1914 y otros (cf. MALKIEL 1976 , p. 757, n. 1).

${ }^{2}$ Por mencionar sólo algunos de los trabajos de MALkiel relacionados con el tema: "Morphological Analogy as a Stimulus for Sound Change", LiS, 4 (1969), 305-327; "Derivational Transparency as an Occasional Co-determinant of Sound Change: A New Causal Ingredient in the Distribution of -cand - $z$ - in Ancient Hispano-Romance", RPh, 25 (1971), 1-52; "Deux frontiers entre la phonologie et la morphologie en diachronie", Lang, 8 (1973), 7987; "New Problems in Romance Interfixation (1): The Velar Insert in the Present Tense (with an Exc. on -zer/-zir Verbs)", RPh, 27 (1974), 304-355; "In Search of Penultimate Causes of Language Change: Studies in the Avoidance of $|z|$ in Proto-Spanish", en Current Studies in Romance Linguistics: Papers from the 1974 Texas Symposium, ed. by M. Luján and F. Hensey, Georgetown University Press, Washington, 1976, pp. 27-36; "Multiconditioned SoundChange and the Impact of Morphology on Phonology", Lan, 52 (1976), 757778; "A Hidden Morphology Factor behind Instances of Erratic Distribution of $c$ and $z$ in Old Spanish?", Lacus Forum, 4 (1978), 511-529; "In Search of Coefficients in Diachronie Morphological Analysis: /i/ as an Increasingly Dominant Vowel in Spanish Inflectional Morphemes", Proceedings of the Annual Meetings of the Berkeley Linguistic Society, 8 (1982), 36-78. 
formal de las unidades lingüísticas - palabras y morfemas con cretamente- sea por cambio fonético, sea por fenómenos morfo fonológicos, y, por otro, la tendencia a su conservación que ga rantizaría la inteligibilidad continuada. La analogía puede esta al servicio de cualquiera de estas fuerzas, la conservadora o 1 i destructora. De esto último sería ejemplo, entre otros, la elimina ción de distinciones gramaticales en Ingvaeónico, según Hock:

In Ingvaeonic, however, loss of $n$ in the context $\mathrm{U}_{-}<+$fric $>$ led to the morphological merger or syncretism of the second an third plural endings; cf. (36). The Ingvaeonic response was a complete eli mination of the distinction between plural endings, by leveling th third/second person ending throughout the plural; cf. e. g. the earl Old English paradigm (37). (Four-part analogy generalized this levelin also to the past tense.)

(36) Ingvaeonic:

$$
\begin{array}{ll}
\text { sg. } 1 \text { bir-u/beor-u } & \text { pi. } 1 \text { *ber-a-m } \\
2 \text { bir-i-s } & 2{ }^{*} \text { ber-a-p } \\
3 \text { bir-i-p } & 3 \text { *ber-a-np > -a-p }
\end{array}
$$

(37) Early Old English:

$$
\begin{aligned}
& \text { sg. } 1 \text { beor-u } \\
& 2 \text { bir-i-s } \\
& 3 \text { bir-i-p }
\end{aligned}
$$

$$
\begin{aligned}
& \text { pl. } 1 \text { ber-a-p } \\
& 2 \text { ber-a-p } \\
& 3 \text { ber-a- } \mathrm{p}^{3}
\end{aligned}
$$

En lo que sigue me concentro sobre todo en las fuerzas conserv: doras. Me interesa identificar su acción en sincronía, en particı lar en la formación de palabras por derivación.

Los recursos para la conservación formal de las palabras y c su estructura interna son múltiples:

1) Evitar la pérdida de algún elemento que por cambio fonét co debería desaparecer. Dworkin menciona por ejemplo que portugués conservaba en ciertas palabras una de las dos cons nantes que por cambio fonético deberían desaparecer:

The course followed in Luso-Romance by VIGILARE 'to ker watch', the source of Ptg. vigiar and velar 'to keep watch, to st: awake', resembles that of RIGIDU. Regular sound change me aced both the -Ǵ- and -L- of the base. As a result the speakers cho

${ }^{3}$ Hock 1988, p. 183; las cursivas son mías. 
to retain one of the two endangered consonants as a therapeutic means of shoring up the phonetic substance of VIGILARE ${ }^{4}$.

y dos años después sostiene que algunas comunidades hispánicas retuvieron la $-d$ - de alauda:

As the tendential deletion of $-d$ - spread through the lexicon, older aloda and the innovative aloa must have coexisted for a time. At the crucial juncture some speech communities dissatisfied with aloa could well have opted to retain the conservative variant with the medial dental intact ${ }^{5}$.

En morfología, tenemos el ejemplo, estudiado magistralmente por Malkiel, de la conservación de $-d$ - en ciertas formas verbales. Después de detectar una variedad de factores que juntos hicieron posible la conservación dice:

Under this set of special circumstances, it becomes for the first time understandable why a large sector of speakers should have clung to the $-d r$ in vide and vido, while freely dispensing with it in such pres. ind. forms as vees, vee, veen. The medial consonant in vide and vido, as against the central cavity in veer, derived support from such triads as crove $_{2}$, crovo 2 /creer; fuxe, fuxo/fuir; raxe, rayo/raer; rise, riso/reir; sove, sovo/seer; traxe, traxo (and vars.)/traer. The process here microscopically observable is an example of paradigmatic resistance to sound shift; the special feature that separates vido from crovo, fuxo, etc. is that the consonant which here temporarily accomplishes the delaying action was the same that was allowed to disappear from other tenses.

y, más adelante:

The most characteristic variecy of the preterital model at issue... protected the $d$ only in rhizotonic forms, juxtaposing vide, vido $\mathrm{y}$ vimos (viemos), vieron, etc. In this accentual restriction Old Castilian bore a remarkable resemblance to Tuscan [...] Conceivably the most cautiously wheighed formulation, in partial improvement upon my earlier statement in $\S 0.3$, would be this: Since, on the one hand, the contrast with respect to $-d$ - between vee $<$ uidet and vido $<$ VIDIT rules out accentual conditions as the sole controlling factor and, on the other, the contrast between vido and vieron discern-

\footnotetext{
${ }^{4}$ DWORKIN 1975, p. 467.

${ }^{5}$ DWORKIN 1977, p. 516.
} 
ibly limits the validity of the claim here staked out for a chara teristically shaped preterital radical, only the chance coinciden -in, at most, two out of six possible forms - of a favorable stre pattern and a distinctively marked tense, was apt to create optim conditions for the preservation of the $-d^{-6}$.

En este caso se trata entonces de la protección que brinda a u elemento fonológico, la $-d$ - intervocálica de las formas de la pr mera y tercera personas singular del pretérito, el paradigma de lc verbos que acentúan estas formas en el radical, favoreciendo a vez la subsistencia del propio paradigma.

2) Cambiar una vocal final de tema de manera que resalte estructura interna de la palabra compleja y el hecho de que se tr: ta de una palabra y no de una frase, por ejemplo: rabilargo, carico tado, grandilocuente, boquiflojo, grandioso, afectuoso (en las palabras d rivadas se hubiera esperado pérdida de la vocal final del tem. pero ésta se conservó disimilada frente a la del sufijo) ${ }^{7}$.

3) Epéntesis de elementos que subrayen la transparencia der vacional. En las estructuras morfológicas es frecuente la aparicić de elementos intercalados en los puntos de contacto entre morf mas. Puede darse por razones fonológicas, como en el caso citac por Malkiel de la variante -güelo de -uelo y -güela de -uela: ala güela, corre-güela, esquir- $(l)$-güe(l)o, etcétera:

Lo peculiar de este grupo es que se trata, dentro de ciertos dialect sociales y regionales, de la sustitución obligatoria de la $\langle w\rangle$ ir cial de sílaba por $\langle\mathrm{gw}\rangle$, es decir, de un cambio rigurosamente $\mathrm{f}$ nológico... ${ }^{8}$

Desde luego este cambio "rigurosamente fonológico" tiene a vez el efecto de marcar la juntura morfológica.

Otros elementos intercalados desde un principio están al se vicio de la morfología. Tienen la función de hacer posible ur formación derivacional, de "llenar un hueco, suavizar una tran ción, aclimatar un préstamo"".

${ }^{6}$ Malkiel 1960 , pp. 315 y 316.

7 También se encuentra la -i- como marca de juntura morfológica en ، rivación. MALKIEL 1958, p. 135 la menciona como una secreción o aglutir ción al sufijo de un elemento o parte de elemento que lo precede y mencior -iano por -ano en ciceron-iano, calderon-iano, galdos-iano, nunes-iano.

${ }^{8}$ Malkiel 1958, p. 165.

${ }^{9}$ Ibid., p. 161. 
Las consonantes antihiáticas:

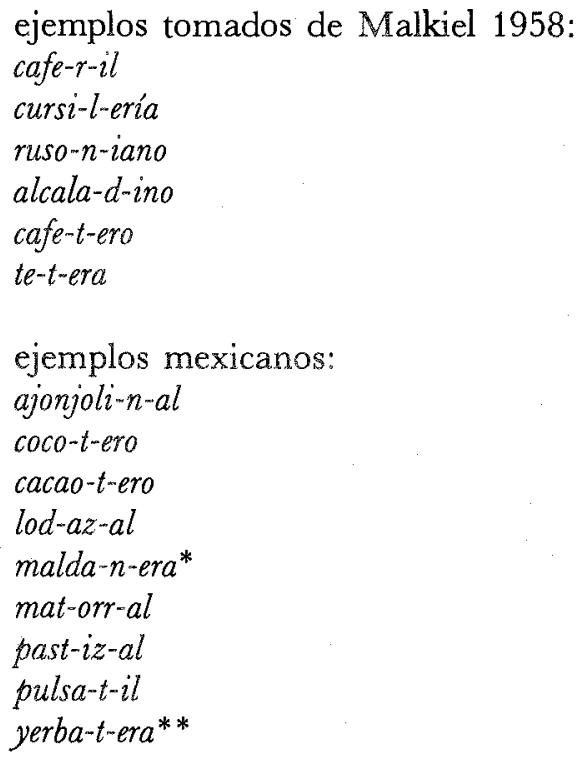

* “ . . a mí esto sí me pesa; pues de estas mujeres no las quiero, porque éstas son maldaneras: le van a hacer la maldad a sus mismos hijos. ¿Yo qué me espero? Yo me voy para mi casa"' (944055014).

** " . . masca uno bastante ajo, habían... de esos que les dicen yerbateros ¿no? (793016176) ('persona que cura con ayuda de hierbas').

Hacen posible las formaciones de las palabras derivadas sin que se pierda la identidad de la palabra base al grado de no poderse interpretar la derivada. Impiden, por ejemplo, la fusión de dos vocales iguales como en *te-era o el paso de hiato a diptongo previsible para otros: *alcalaino $>{ }^{*}$ alcalaino, etcétera.

En español, la regla general es que se pierdan las vocales finales ante un sufijo que comienza con vocal. No sucede así, sin embargo, cuando la vocal final es tónica. Suele entrar entonces a prestar servicio alguna consonante antihiática: cafe-r-il. También se puede proteger así una vocal final que se considera importante para la identificación de la base como la que encontró Malkiel en francés, por ejemplo en los derivados de apellido: hugolien, hugolesque, hugolatrie ${ }^{10}$ :

10 Malkiel 1958, n. 100. 
In the closest vicinity of interfixes one may place those "attach ments" that obviate the need for apocopating such final vowels 0 primitives as seem entitled to special consideration, because the pertain to proper names or to exotic words, or else because, counte to the general trend, they are stressed, especially if they happen $t$ mark the peak of a monosyllable, cf. Fr. hugo-l-ien, -esque besid (Victor) Hugo, Ptg. cha-l-eira 'teapot' beside chá 'tea'11.

O, en español, el ya citado cacaotero donde se evita un grupo vocá lico triple o un parecido fonético indeseable; o las palabras cultas eritema-t-oso, esperma-t-oso, granuloma-t-oso.

Frecuentemente se intercalan elementos cuando hay grup vocálico final, con o sin pérdida de una de las vocales. Document bacaladero de bacalao (relacionado tal vez con ultracorrección). E frecuente la vacilación con diptongos finales y así documento po ejemplo turbiedad pero distribumiento.

He encontrado las siguientes soluciones a la formación d abstractos de cualidad en -ad a partir de palabras terminadas e las secuencias vocálicas (io, ia, io, ia, ua, uo):

-io, -ía

1) añadir -dad con o sin ayuda de un interfijo fri-al-dad

2) añadir -edad después de perder la última vocal vaciedac piedad.

$-i o,-i a /-u o,-u a$

1) añadir -edad después de perder diptongo: necedad;

2) añadir -edad después de perder la vocal final: turbiedad, ebri dad, antigüedad. Esta última es la solución favorecida, lo que sugit re que se creó este alomorfo a partir de -idad por disimilación para conservar la transparencia de la palabra.

3) añadir -idad después de perder la vocal final: contiguo/cont güidad.

Otra de las funciones de los elementos intercalados es evit: la creación de homónimos de palabras ya existentes. Al intercalí interfijos se posibilita la creación del derivado que de otra manes hubiera quedado bloqueado por un homónimo existente en el 1 . xico. Frecuentemente parece que el elemento intercalado cump ambas funciones: la morfológica de conservar la transpares

11 Malkiel 1970, p. 320. 
cia y la léxica de evitar homonimias y homofonías; tal vez sean ejemplos, entre otros, aguacero vs. agüero, alcaladino vs. alcalino.

Donde el interfijo surgió como residuo y ha servido posteriormente para diferenciar dos elementos léxicos, esta función diferenciadora le garantiza su permanencia: pan-ad-ero vs. panero; carnic-ero vs. carnero, mencionados también por Malkiel ${ }^{12}$. Entre otros, el interfijo le garantiza un acento secundario a la base. Esto y el mayor volumen le da a ésta la prominencia deseada: fort-aleza, fort-al-ecer; for-ast-ero.

4) Cruces y contaminación. Un elemento cuya identidad formal está muy amenazada o cuya forma contraviene tendencias fonológicas de la lengua a la cual pertenece, ocasionalmente puede sobrevivir en un cruce con un cuasi-sinónimo que, por así decirlo, le presta cuerpo fónico.

Dworkin, después de aludir al rechazo del español antiguo a los grupos vocálicos áo, óa úo, úa y de mencionar algunos recursos empleados por los hablantes para evitarlos (epéntesis, supletivismo), dice:

Other groups of speakers attempted to strengthen aloda or to reshape aloa by adopting the -ndr- cluster of the (near) synonym calandria (or of golondrina, if Corominas' hypothesis holds water). In the long run this experiment proved successful, as its product alondra, became the usual designation for the lark in the literary language ${ }^{13}$.

5) Préstamos foráneos, supletivismo culto. En algún momento la comunidad hablante se rinde ante la imposibilidad de conservar una forma o de hacer viable una formación que garantice significantes diferentes para significados distintos.

Cuando todo parece fallar y la pérdida del elemento se ve inevitable, sea por cambios aplicables, sea porque cambios ya ocurridos dejaron una forma inaceptable, se suele optar por un préstamo ${ }^{14}$, si se trata de una forma libre y por el supletivismo culto, cuando se trata de una forma ligada.

Como ejemplo del destino de un elemento libre sirve nuevamente el caso de alauda, pero en portugués:

12 Malkiel 1958, p. 166.

${ }^{13}$ DWORkin 1977, p. 517.

${ }^{14}$ Desde luego se trataría sólo de una razón entre muchas para adoptar préstamos. 
In the passage from Latin to Luso-Romance, ALAUDA was ex posed to the elision of both - L- and -D-. The danger of excessivt phonetic erosion could very well have doomed ALAUDA, as thi speech community was hesitant to tolerate such a form as *ao(u)a Instead of attempting to salvage the local progeny of ALAUDA through therapeutic retention of one of the endangered consonant (as in rijo "strong" < RIGIDU or vigiar $\infty$ velar "to keep watch stay awake" < VIGILARE) or through insertion of an antihiati $v$ after ou < AU (as in ouvir OPtg oir "to hear" < AUDİRE) speakers turned to the available Germanic substitute ${ }^{15}$.

Malkiel detecta un hecho similar en francés aunque los mo tivos son distintos: "El francés aprovecha un préstamo germá nico (boulanger) para no dejar semánticamente hipertrofiado pan ier'"16.

El préstamo aparece así como estación final de un viaje po diversos intentos de conservación o de construcción de un deriva do. El supletivismo culto en derivación es muy frecuente y un de sus orígenes es la dificultad para formar derivados decoroso a partir de la palabra vernácula, entre otras cuando ésta es mu breve: luz: luminoso; oír: audible, audición; pobre: pauperismo (DEM) o por presentar alguna otra dificultad, como cuando la previsibl reducción de un diptongo oscurece la base y le resta cuerpo: fiei fidelidad, niebla: nebuloso, nebulización (DEM). El supletivismo cult puede también tener la función de diferenciar elementos léxicos campo: campismo, campo: agrarismo.

6) Préstamo vernáculo. Me interesa colocar en este context un fenómeno que he observado en mi material (analizo las pala bras derivadas del corpus del Diccionario del Español de Méxicc $D E M)^{17}$ y que considero muy relacionado con lo que anteced el uso de bases supletivas vernáculas para hacer posible una der: vación; por ejemplo, en patada (que proviene de pie, pues la pataa se da con el pie) o maternidad (semánticamente relacionado co madre, pero formado sobre el tema del adjetivo materno).

Malkiel explica que se habla de interfijos cuando el tema rı sultante de la palabra contenida más el interfijo no existe com tema independiente en la lengua:

${ }^{15}$ DWORKIN 1955, p. 517.

${ }^{16}$ Malkiel 1958, n. 107.

17 Trabajo con el corpus del Diccionario del Español de México, El Colegio c México, que corresponde a 2 millones de palabras en texto. 
Es el segmento siempre átono y falto de significado propio, entre el radical y el sufijo de ciertos derivados, p. ej. el elemento -ar en hum-ar-eda, polv-ar-eda, palabras que no es lícito descomponer en humar- y polvar-eda, por no existir ni haber existido nunca, que sepamos, las fases intermedias *humar, *polvar como formaciones independientes ${ }^{18}$.

Más adelante dice que los interfijos son casi siempre sufijos truncos y desemantizados: "En la gran mayoría de los casos, el interfijo equivale, por las consabidas causas históricas, a una variante truncada de un sufijo" 19 .

Entiendo que estos sufijos están apareciendo en contextos distintos al de los elementos léxicos libres. Es decir, que por lo menos en el estado de lengua contemplado no existe la base tema + sufijo como forma libre aunque el sufijo pleno pueda aparecer en otras palabras en el léxico de esa época.

El deslinde con respecto a las formaciones que voy a mencionar en seguida es claro: mis ejemplos están constituidos por palabras derivadas que sí existen en la lengua contemporánea de las formaciones "secundarias". Lo que tienen en común con los interfijos, es que también están desemantizadas; es decir, se trata de préstamos de la pura forma de una palabra derivada de la propia lengua. Se puede hablar de supletivismo vernáculo, aunque este término no se aplicaría del todo, puesto que en las formaciones supletivas suele conservarse el significado. Se toma el signo completo en vez de aquél que por alguna característica no resulta viable.

Aquí, en cambio, una forma, por lo general de la misma familia de palabras, aunque puede también tener únicamente parecido formal con la base pretendida, funge como base de una derivación, prestando un radical alargado al procedimiento ${ }^{20}$. Función y efecto son los mismos de la introducción de interfijos: se hace viable una formación derivacional. La relación de la pa-

18 Malkiel 1958, p. 107.

19 Ibid., p. 144.

${ }^{20}$ No debe confundirse este procedimiento con aquél en que también se utiliza como base una palabra que no aparece en la definición, pero que está como representante por excelencia de lo que se pretende denotar con la base. Éste sería el caso de alpinismo 'deporte de montaña' o 'de escalar montañas' y no 'deporte de escalar los Alpes', donde, sin embargo, figura Alpes con todo su significado y poder denotativo sólo que en tanto designa un prototipo de montaña. 
labra derivada secundaria se establece con la primitiva, y el sufijc de la derivada primaria queda como interfijo desemantizado. La diferencia estriba en que la derivada primaria contenida sí existe como elemento léxico independiente.

Así voluntarioso semánticamente remite a voluntad; es voluntario. so el que muestra voluntad para hacer algo, el que tiene buenć disposición o el que hace algo a base de fuerza de voluntad. Nc está contenido aquí voluntario más que formalmente. Tampocc corresponde esta formación al esquema de los adjetivos postadje tivos en -oso que pueden en su mayoría parafrasearse con 'qu tiende a X' 'que es un poco X' verdoso, amarilloso (cf. blanquecino blancuzco, rojizo), maloso. Tembloroso contiene temblor, sin embargo semánticamente remite al verbo temblar y tiene por significadı 'que tiembla' (cf. lloroso 'que llora, ha llorado o está a punto d' llorar'). Seguramente se tomó la forma temblor por incomodidar con tembloso.

Pasajero, como adjetivo y como sustantivo, se relaciona má fácilmente con el verbo pasar: pasajero, - $a_{\mathrm{aj}}$ 'que pasa pronto', pa sajero $_{\text {st }}$ 'que se traslada ("pasa') en vehículo, de preferencia de transporte público', aun cuando el pasajero suele pagar "pasa je"'. *Pasero era muy corto y el posparticipial existe, por lo menc en México, con otro significado: pasadero 'que pasa por bueno' el posgerundio está reservado a denominaciones de oficios (lavar dero, curandero, rezandero, cuidandero); en todo caso los adjetive posverbales en -ero no se forman a partir de gerundios sino de radical de participio: duradero, valedero, pasadero, pagadero, así qu se recurrió a un radical prestado de pasaje en una demostració de solidaridad familiar.

Tabacalero, - $a$, aparte de tener función diferenciadora frente tabaquera como ya señaló Malkiel ${ }^{21}$, contiene tabacal, palabra qu sí existe en el español contemporáneo, pero que está contenic sólo formalmente: en "(industria) tabacalera" el significado remi a tabaco y no a tabacal.

Tal vez sea algo distinto el caso de callejero, donde origina mente se hubiera formado el derivado sobre calleja, pero hoy asociación se hace con calle por haber caído en desuso esa pal bra. Aun así el tema derivado primario existe de manera ind pendiente en la lengua actual: callejón, sin que callejero se relacior por su significado particularmente con los callejones.

Colonialismo se relaciona semánticamente con colonia o color

21 Malkiel 1958, n. 107. 
zación antes que con colonial; es el afán de un estado o de sus habitantes de ver a otros estados como sus colonias, o el afán de colonizar. La presencia del elemento - k garantiza la aparición completa de colonia a pesar del diptongo final.

Finalidad y penalidad, ambos derivados sin novedad semántica frente a alguna acepción de fin y pena, se relacionan con estas palabras y no con lo final o lo penal. Igualmente divinidad no es la cualidad de lo divino ni festividad la cualidad de lo festivo ni lo festivo mismo, divino y festivo son préstamos de la propia familia de dios y fiesta para crear términos más largos para referirse a éstos.

Finalmente, maternidad y paternidad, si son herencia directa del latín, en sincronía se interpretarían sin embargo como derivados de madre y padre respectivamente y no de materno, maternal ni paterno, paternal; puesto que su significado es 'condición de madre' y 'condición de padre (físico o, por ejemplo, intelectual, cuando se trata de una idea). Pueden interpretarse por lo tanto también como préstamos formales de la propia familia.

El interpretar estas palabras como derivadas formalmente del derivado primario o como creadas por contaminación con éste acaba siendo cuestión de criterio del estudioso. En lo personal opto por verlos como bases prestadas a la propia lengua, proceso que me parece estar documentado también en creaciones coloquiales festivas como: frijolito por frio: "Hace frijolito" o cuaderno por cuate o como el ejemplo que me sirve de subtítulo.

Elisabeth Beniers

Universidad Nacional Autónoma de México

\author{
BIBLIOGRAFÍA
}

Dressler, WolfGang U. 1977. Grundfragen der Morphologie. Verlag der Oesterreichischen Akademie der Wissenschaften, Wien.

Dworkin, Stephen N. 1974-75. "Therapeutic Reactions to Excessive Phonetic Erosion. The Descendants of RIGIDU in Hispano- and LusoRomance", $R P h, 28,462-472$.

- 1977. "Therapeutic Reactions to Phonotactic Awkwardness. The Descendants of alauda in Hispano-Romance", $Z R P h, 93,513-518$. 
Hooper, JoAn B. 1976. An Introduction to Natural Generative Phonology. Aca demic Press, New York.

MALKIEL, YAKOV 1958. "Los interfijos hispánicos", HAM, 2, 107-199.

—_ 1960. "Paradigmatic Resistance to Sound Change: The Old Spanis] Preterite Forms vide, vido against the Background of the Recession of Pri mary -d-", Lan, 36, 281-346.

- 1976. "Multi-conditioned Sound Change and the Impact of Morphol ogy on Phonology", Lan, 52, 757-778.

Martinet, André 1965. "De la morphologie", LingP, 1, 15-30.

Richardson, John F. et al. 1983. Papers from the Parasession on the Interplay c Phonology, Morphology and Syntax. University of Chicago Press, Chicago.

Rochet, Bernard 1974. "A Morphologically Determined Sound Change i Old French", Ling, 135, 43-56.

Rudes, B. A. 1980. "On the Nature of Verbal Suppletion", Ling, 18, 655 676.

Stankiewics, Edward 1962. "Interdependence of Paradigmatic and Derive tional Patterns", Word, 18, 1-22. 